\title{
KNOWLEDGE AND UTILIZATION LEVELS OF PARTOGRAPH IN MANAGEMENT OF LABOUR AMONGST MIDWIVES IN SELECTED HEALTH FACILITIES IN AKURE, ONDO STATE
}

\author{
Adamolekun Patience Arinola', Osaji Teresa ${ }^{\mathbf{2}}$ and Adeyanju Awoniyi Babafemi ${ }^{1}$ \\ ${ }^{1}$ Faculty of Nursing, Niger Delta University, Wilberforce Island, Bayelsa State \\ ${ }^{2}$ Faculty of Nursing, University of Calabar
}

Cite this article:

Adamolekun P.A., Osaji T., Adeyanju A.B. (2021), Knowledge and Utilization Levels of Partograph in Management of Labour Amongst Midwives in Selected Health Facilities in Akure, Ondo State. African Journal of Health, Nursing and Midwifery 4(6), 101-118. DOI: 10.52589/AJHNMFGRZBGWS.

\section{Manuscript History \\ Received: 16 Nov 2021 \\ Accepted: 1 Dec 2021 \\ Published: 14 Dec 2021}

Copyright $\odot 2020$ The Author(s). This is an Open Access article distributed under the terms of Creative Commons AttributionNonCommercial-NoDerivatives 4.0 International (CC BY-NC-ND 4.0 ), which permits anyone to share, use, reproduce and redistribute in any medium, provided the original author and source are credited.

\begin{abstract}
Routine use of Partograph prevents labour related deaths which contributes hugely to maternal mortality in SubSaharan Africa (WHO, 2015).The study assessed knowledge and utilization levels of partograph in management of labour amongst midwives in selected health facilities in Akure, Ondo State. A cross-sectional descriptive survey design was adopted. Two hundred respondents were selected using a multistage sampling method. A validated self-structured questionnaire was used for data collection after a test retest for reliability. Data were analyzed using descriptive statistics of frequency, means and percentages while inferential statistics of Chi-square ANOVA was used to test hypothesis at significance of $P \leq 0.05$. The result showed that the majority of the midwives 119 (59.5\%) had good knowledge of partograph. Also, on levels of utilization, 62(62\%) of respondents from UNIMEDTH and 46 (59\%) in MCHA had good utilization of partograph, while all the 22(100\%) respondents in CHCA had poor utilization. There was a significant relationship between years of work experience and midwives' level of knowledge of partograph $(p=0.01)$. Annual partograph utilization rate was highest in the tertiary facility (UNIMEDTH (63\%), followed by secondary (MCHA 51.8\%) and primary (CHCA 41.3\%). In conclusion, although the respondents had good knowledge of partograph, its utilization level was low at CHCA, therefore practical skills, training and retraining programs for midwives should be instituted to enhance the level of utilization of partograph in the primary health care facilities. Further study for midwife-led intervention on the utilization of partograph among midwives in the PHC should be done at a wider scope.
\end{abstract}

KEYWORDS: Labour, Maternal Mortality, Midwives, Partograph, Utilization 


\section{INTRODUCTION}

Maternal death is a global phenomenon that is worrisome, and approximately 300,000 maternal deaths occurred globally in 2013 of which 98 percent occurred in developing countries (Yisma, Dessalegn, Astatkie, \& Fesseha, 2013). On average, 230 women die per every 100,000 live births each year in developing countries (Yisma, Dessalegn, Astatkie, \& Fesseha, 2013). Global estimates from World Health Organization (WHO) in 2015 showed that there were 216 maternal deaths per 100,000 live births, of which about 99 percent accounts for maternal deaths in developing regions with Sub-Saharan Africa accounting for roughly 66 percent. One major cause of maternal mortality is obstructed labour which usually results from neglected prolonged labour.

Evidence abounds that cost-effective and affordable health interventions like the use of the partograph will contribute to curbing the alarming number of maternal deaths, especially intrapartum maternal deaths (Sama, Takah, Danwe, Melo, Dingama, \& Angwafo, 2017). Partograph is a very useful graphical record of the course of labour that yields optimum results when employed in labour management by obstetric caregivers (Asibong et al., 2014). Following recommendations from WHO, the routine use of partograph to monitor the progress of labour in all settings is encouraged and promoted particularly via the maternal and neonatal health programs (WHO, 2015). Therefore, obstetric care providers (OCPs) at all levels of care are expected to know how to use and interpret the partograph. However, even though partograph is a simple and inexpensive tool which has been proven to effectively prevent maternal deaths and averts complications due to obstructed or prolonged labour, it is not as widely implemented as it should be (Abebe, Birhanu, Awoke \& Ejigu, 2013).

The partograph is used in the clinical context and the potential impacts of its effectiveness have led to the realistic systematic review of partograph use (Bedwell, Levin, Pett, \& Lavender, 2017). It acts as an early warning system, it alerts care providers to deviations from normal progress, it indicates when a woman requires emergency intervention, such as referral to a higher-level facility, labour augmentation or cesarean section (CS) (Mathibe-Neke, 2013). In developed nations, partograph is an obstetric tool that is useful and efficient in monitoring labour and according to literature the acquisition of knowledge of its use and proper application of that knowledge would culminate in a remarkable reduction in the incidence and negative outcomes of prolonged and obstructed labour, which is reported to be associated with 10 percent of maternal deaths (Tadesse, 2012). The women who experience obstructed labour usually suffer from postpartum haemorrhage, uterine rupture, puerperal sepsis, and obstetric fistula (Dolea \& AbouZahr 2003).

Furthermore, obstructed labour is highly associated with birth trauma, birth asphyxia, stillbirths, neonatal sepsis, and neonatal deaths, thus, prevention of obstructed and prolonged labour using partograph during labour is a key intervention in the reduction of maternal and perinatal morbidity and mortality (Wakgari, Amano, Berta, \& Tessema, 2015). In Southeast Asia, a study by WHO (2011) reported that the use of the partograph following standard protocol reduces prolonged labour from 6.4 percent to 3.4 percent, the proportion of labour requiring augmentation from 20.7 percent to 9.1 percent, emergency caesarean section from 9.9 percent to 8.3 percent, and stillbirths from 0.5 percent to 0.3 percent. Therefore, proper use of a partograph in an environment where referral and timely intervention are not possible would greatly contribute to the reduction of maternal morbidity and mortality in the region (WHO, 2011). Furthermore, Sama et al., (2017) mentioned that, proper utilization of the partograph 
following standard protocol, with keen attention to the alert and action lines, allows for timely identification and diagnosis of pathologic labour and thus aid in guiding timely decisionmaking regarding the necessary interventions.

The focus of using the partograph in developing countries is on the prevention of maternal and fetal morbidity and mortality related to prolonged and obstructed labour (Asibong et al., 2014). Additionally, it has been reported from a study in Nigeria that Partograph use in obstructed labour management enhances maternal and neonatal well-being (Asibong et al., 2014). A study conducted by Yisma et al., (2013), in Addis Ababa, Ethiopia indicated that, over half (57.3 percent) of the obstetric care givers reported use of the modified WHO partograph to monitor women in labour revealed that midwives had poor knowledge and utilization of Partograph.

Knowledge is a prerequisite to use of partograph through intensive teaching of midwifery staff before implementation of the partograph (Engender health, 2012). According to Mathibe-Neke, (2013), it was revealed that 13 midwives (38percent) reported that they had never received any training on the use of partograph while 11percent had been attending training on the use of partograph for more than two years. This result signifies a limited number of midwives with adequate knowledge on the use of partograph.

Therefore, the study ascertained the knowledge and utilization levels of partograph in management of labour amongst midwives in selected health facilities in Akure, Ondo state.

\section{Statement of the Problem}

Mortality rate remains a major public health problem especially in developing countries like Nigeria where the maternal mortality rate is 917 deaths per 100,000 live births making it the second country with the highest maternal mortality rate after Sierra Leone that has 1,120 deaths per 100,000 live births. Ghana has a rate of 308 deaths per 100,000 live births, while Sudan rate is 295 deaths per 100,000 live births and Rwanda has 248 deaths per 100,000 live births (WHO, 2015). The World Health Organization (WHO) recommends using the partograph to follow labour and delivery, with the objectives to improve health care and reduce maternal and foetal morbidity and death (WHO, 1994). Hence, the use of partograph was adopted as one of the methods to reduce maternal mortality in Nigeria and to meet the Goal-3 target of the Sustainable Development Goals (SDGs) by 2030 in line with reducing the global maternal mortality ratio to less than 70 per 100,000 live births (Yisma et al., 2013).

In order to meet up the above stated target, the Nigerian government developed a policy to end all preventable maternal deaths by training and retraining midwives on the utilization of partograph. The government went a step further in making partograph forms available to all health institutions including primary healthcare centers. Yet, available studies have revealed poor utilization of the partograph chart which does not seem to correspond to the wealth of knowledge among nurse-midwives (Fawole, Hunyinbo \& Adekanle, 2008; Lavender, Omoni, Lee, Wakasiaka, Watiti \& Mathai, 2011).

During a survey carried out in 2008 by the Federal Ministry of Health Nigeria and the United Nations Fund for Population Activities, a health worker stated "we don't know what a partograph is. We have not seen it, we have only heard of it" There is no national data on the availability and utilization of partographs in all health facilities (FMOH \& UNFPA, 2008). With the reports available, there seems to be a conflict of information concerning the level of knowledge on partograph chart availability and utilization in Nigeria. Hence it is necessary to 
assess the knowledge and the utilization levels of partograph in the management of labour amongst midwives in selected health facilities in Akure, Ondo State.

It was observed that, most health care institutions do not enforce the use of the partograph chart to monitor parturients; there are no regular training programs on partograph for midwives and other birth attendants; and no policy guideline as protocol for the use of partograph in all the healthcare delivery centers (Fawole et al,2008).

Also, during an interactive session with some of the student-midwives from the Ondo State School of Midwifery who were posted to various health facilities for their Obstetrics \& Gynaecology clinical posting; it was gathered that the partograph charts were hardly used to monitor labour even among the chief matrons, while those who use the chart do not observe protocol nor document properly.

Research has shown that the use of a partograph to manage labour improves maternal and newborn survival and reduces the need for additional interventions, such as caesarian section (Bosse, Massawe \& John, 2002). Furthermore, it was reported that the use of partograph was more frequently used in tertiary level of care compared to primary and secondary level of care in monitoring parturients by midwives (Fawole et al,2008). However, there is yet no study (as at the time of this study) that compares knowledge and utilization levels of the partograph among the midwives working in the primary, secondary and tertiary healthcare delivery levels in Akure, Ondo State.

Hence, this study is pertinent to assess the knowledge and utilization levels of partograph in management of labour amongst midwives at selected health facilities in Akure, Ondo State.

\section{Research Questions}

1. What is the level of knowledge on Partograph in the management of labour amongst midwives in selected Health facilities in Akure Ondo State.?

2. What is the level of utilization of Partograph in the management of labour amongst midwives in selected Health facilities in Akure, Ondo State.?

\section{Research Hypotheses}

1. There is no significant relationship between midwives' years of work experience and their level of knowledge on Partograph in the management of labour in selected health facilities in Akure, Ondo State.?

2. There is no significant difference between the level of utilization of Partograph and the midwives' health facilities in the management of labour in Akure, Ondo State.? 


\section{LITERATURE/ THEORETICAL UNDERPINNING}

\section{The Concept of Partograph}

Maternal mortality is unacceptably high; about 830 women die from pregnancy or childbirthrelated complications around the world every day. It was estimated that in 2015, roughly 303,000 women died during and following pregnancy and childbirth worldwide of which 99 percent occurred in developing countries (WHO, 2015). Between 1990 and 2015, maternal mortality worldwide dropped by about 44 percent. Between 2016 and 2030, as part of the Sustainable Development Goals (SDGs) the goal 3 target is to reduce the global maternal mortality ratio (MMR) to less than 70 per 100,000 live births (WHO, 2016). The Partograph is the most used labour monitoring tool, widely supported by health professionals and recommended by WHO for use during labour. Maternal mortality resulting from prolonged and obstructed labour is preventable and there are convincing reports that acquisition of adequate knowledge and proper utilization of the Partograph would culminate in a remarkable reduction in its incidence, which currently constitutes about 8-10 percent of maternal deaths (Asibong et al., 2014).

The partograph consists of four (4) parts as follows;

1. The patient's information, this consists of name, gravid, para, hospital number, date of admission and time of ruptured membranes or time elapsed since rupture of membranes.

2. The fetal condition, observations charted are the fetal heart rate, state of membrane, liquor and moulding of the fetal skull.

3. The progress of labour, observations charted are the cervical dilatation, descent, uterine contractions drugs and intravenous fluids which include the oxytocin regimen.

4. The maternal condition, observations charted are pulse, blood pressure, respiration, temperature and urine test volume, presence of glucose, protein and acetone if any (Rakotonirina et al., 2013).

Therefore, obstetrics care providers (OCPs) at all levels are expected to know how to correctly use and interpret the partograph, which will in turn significantly reduce the sequela of maternal and fetal wastages (Sama et al., 2017). Lavender, Omoni, Lee, Wakasiaka, Watiti and Mathai, (2011) opined that although the partograph is a simple and inexpensive tool which has been proven to effectively prevent maternal and neonatal deaths and the ensuing complications due to obstructed or prolonged labour, it is not as widely implemented as it should be.

\section{Theoretical Framework}

For this study Knowledge, attitude and practice (KAP) theory was used, propounded by Badran (1995) and the Behavioural model by Anderson. The KAP theory constitutes a triad of interactive factors characterized by dynamism and unique interdependence. Emphasis is laid, for each component of the triad, on the value of ethical conduct in raising the application of the component in real life to a peak. Special treatment is given to several important factors that can influence or control the course of practice in the medical profession. An in-depth consideration is given to the conglomerate of information, education, communication, and human resources development, the socio humanistic elements of practice, modern technologies and technology 
transfer system, environment factors and the capability of science and technology for forecasting and assessment. Knowledge is the capacity to acquire, retain and use information; a mixture of comprehension, experience, discernment and skill.

Attitude refers to inclinations to react in a certain way to certain situations; to see and interpret events according to certain predispositions; or to organize opinions into coherent and interrelated structures. While practice means the application of rules and knowledge that leads to action and good practice is an art that is linked to the progress of knowledge and technology and is executed in an ethical manner. According to Badran (1995), the triad of knowledge, attitude and practices in combination governs all aspects of life of human societies and all three pillars together to make up the dynamic system of life itself. It is in this context that ensuing discussion builds up a thesis which focuses on the three pillars and their relevance to health, service and medical care.

\section{Application of KAP Theory to Knowledge and Utilization of Partograph}

\section{Knowledge}

The understanding of midwives on partograph determines their attitude towards its use Midwives with adequate and correct information and awareness about partograph will be able to get involved and have a positive attitude towards its uses in care of a woman in labour. Knowledge in general grows through public education. Therefore, midwives should be well informed and educated on partograph, its uses and importance of its utilization. Adequate information should be disseminated to midwives either through seminars, workshops or in various nursing institutions on partograph.

\section{Attitude}

This indicates thought and point of view about the partograph. Midwives with adequate knowledge on partograph and its importance in the management of labour will surely have an encouraging attitude towards it. Midwives should be made to see and make a view of the positive aspect of it and this can only be done by adequate information during training and motivation should also take place.

\section{Practice and Use}

This is the act of participating, using and applying the knowledge of partograph. Midwives who have good knowledge of partograph and its importance will be involved and also encourage/ teach others on how to use it in reducing maternal mortality rate related to prolonged and obstructed labour by proper monitoring of the fetal conditions such as fetal heart rate, descent of the head and state of moulding in order to know if the labour is progressing and also to prevent prolonged labour. Proper use of it will also help to know when there is obstructed labour to enable the midwife to take appropriate action. Therefore, midwives should be well educated on how to utilize it. 
African Journal of Health, Nursing and Midwifery

ISSN: 2689-9418

Volume 4, Issue 6, 2021 (pp. 101-118)

www.abjournals.org
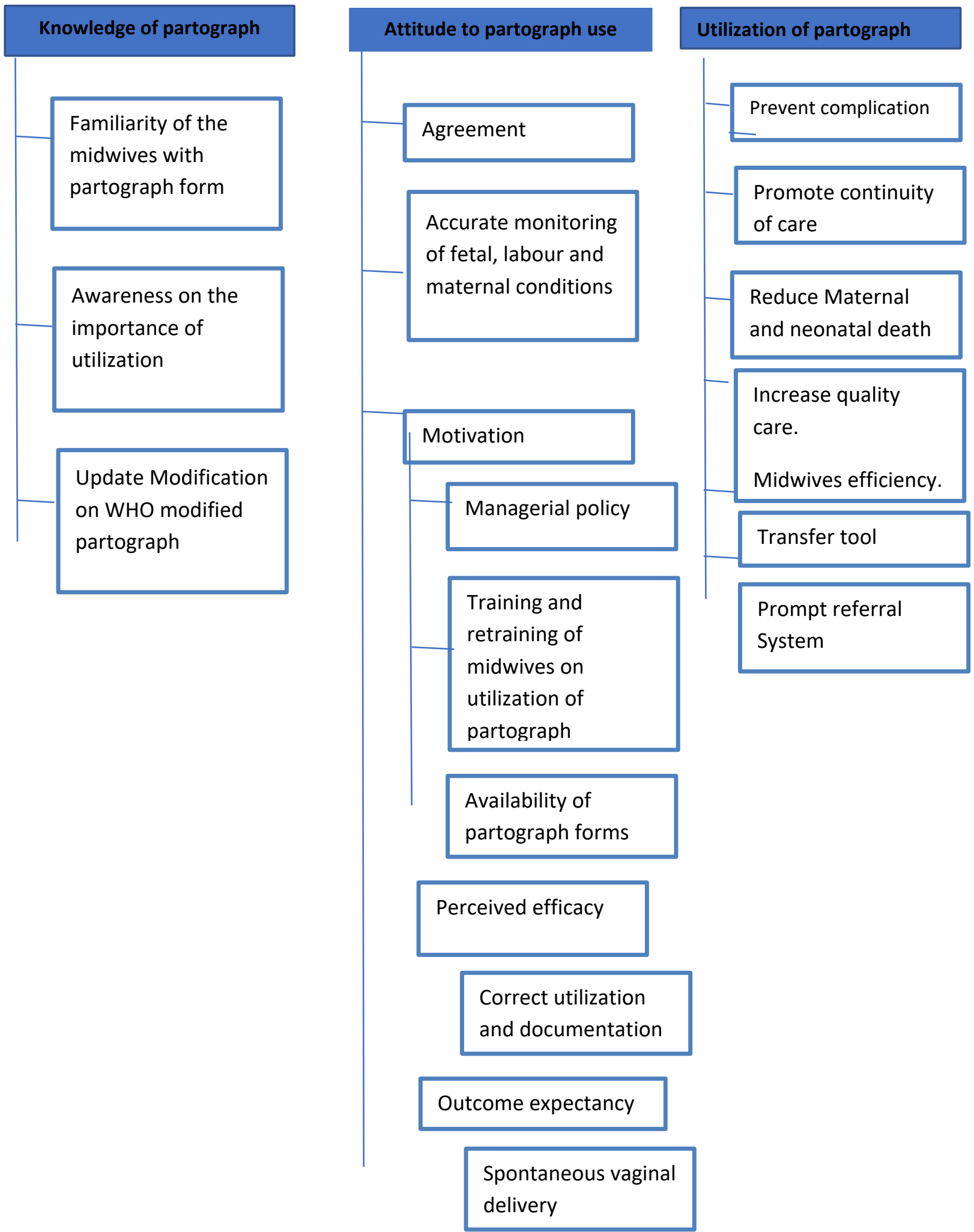

Fig 1: Conceptual Framework

Adapted from KAP model (Badran, 1995) 


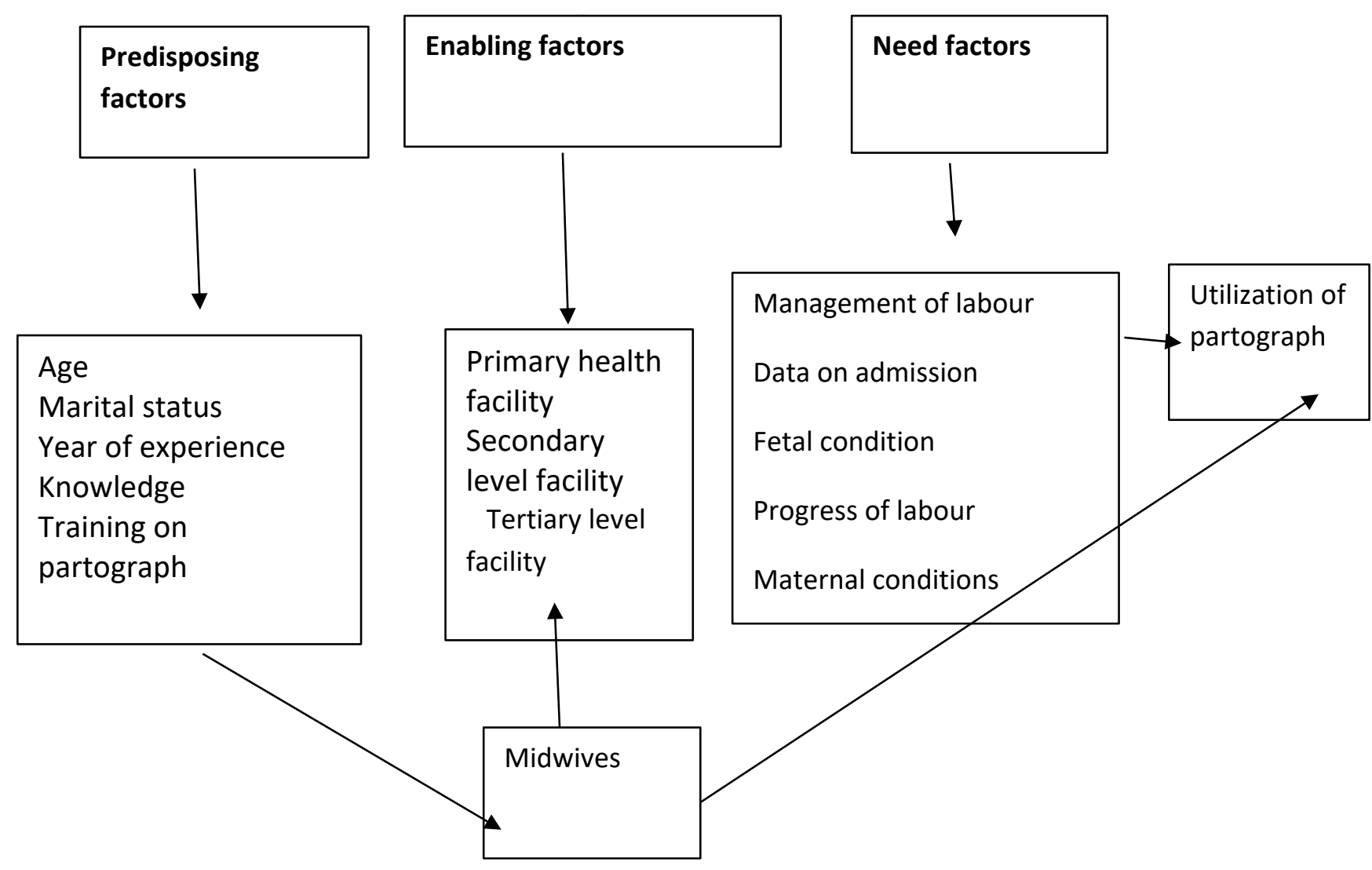

Fig 2: Conceptual Model for Utilization of Partograph Adapted from Anderson Behavioral model (Anderson, 1995)

\section{METHODOLOGY}

\section{Research Design}

The study adopted the cross-sectional descriptive design to ascertain the knowledge and utilization levels of partograph in the management of labour amongst Midwives in selected healthcare facilities.

\section{Target Population}

The target population for the study was the midwives working in the Obstetrics and Gynecology Departments of the hospitals irrespective of their level in the healthcare system. According to the staff statistics from the Obstetrics and Gynecology Departments of CHCA, MCHA and UNIMEDTH, the number of midwives were 22, 78 and 100, respectively. Therefore, the total target population was 200. Details are shown in Table 1 below: 
Table 1: Distribution of Midwives across Selected Healthcare Facilities ( $N=200)$

\begin{tabular}{llll}
\hline WARD & CHCA & MCHA & UNIMEDTH \\
\hline Antenatal ward & 4 & 16 & 22 \\
Labour Ward & 8 & 16 & 18 \\
Clinic & 2 & 12 & 13 \\
Neonatal & 4 & 12 & 14 \\
Post-natal & 4 & 12 & 16 \\
SCBU & - & 10 & 17 \\
Sub total & $\mathbf{2 2}$ & $\mathbf{7 8}$ & $\mathbf{1 0 0}$ \\
\hline
\end{tabular}

\section{Sample Size}

The sample of this study was made up of all the 200 midwives working in the Obstetrics and Gynecology Departments CHCA, MCHA and UNIMEDTH. The entire population units were selected as the sample because of the relatively small size of the population.

The criteria for inclusion were:

1. Willingness to participate in the study

2. Must be a midwife working in the Obstetrics and Gynaecology Department of the selected healthcare institutions.

\section{Sampling Technique}

Multistage cluster sampling technique was used, in two steps: firstly, healthcare facilities were selected using purposeful sampling techniques as used by Ashibong et al (2014). Secondly, total enumeration was used to select eligible respondents due to the relatively small size of the population (200 midwives).

Table 2: Study Sample Size Distribution

\begin{tabular}{|l|l|l|l|}
\hline S/N & HealthCare Facilities & Population & Sample \\
\hline $\mathbf{1}$ & UNIMEDTH & 100 & 100 \\
\hline $\mathbf{2}$ & MCHA & 78 & 78 \\
\hline $\mathbf{3}$ & CHCA & 22 & 22 \\
\hline
\end{tabular}

\section{Instrument for Data Collection}

A self-structured questionnaire was utilized to obtain data to answer research questions 1,2 and 3. The questionnaire had sections A, B, C and D. Section A elicited demographic data; Section B which is scaled elicited information on midwives' knowledge on the use of partograph, section $\mathrm{C}$ used an adapted questionnaire on the use of partograph (Zelellw \& Tegegne, 2018) while Section D provided information on factors affecting utilization of partograph among midwives. Section B consists of questions with True or False responses. The maximum attainable score was 18 while the minimum was 0 , the level of knowledge was graded into "poor knowledge" $(\leq$ Mean Score $=15.2)$ and "Good knowledge" $(\geq$ Mean Score 
15.2). Section $C$ consists of questions with Yes or No responses. The maximum score of 2 and minimum of 0 for each section with a total score of 28 . The Mean score was 14.29 , any score below the mean of 14.29 indicated good utilization, while the score above the mean of 14.29 indicated poor utilization.

Section D consisted of 4 level Likert scale, it contained 9 structured questions which extracted data on factors affecting the utilization of partograph amongst midwives in the management of labour in the selected health care facilities. Respondents ticked $(\sqrt{ })$ "'Strongly Agree - SA, Agree -A, Disagree-D and Strongly Disagree - SD" as many options as applied to them. The responses were weighted $4,3,2$ and 1 , respectively. The maximum attainable score was 36 while the minimum attainable score was 9 .

\section{Validity of the Research Instrument}

It was pretested to establish face and content validity as according to Burns et al. (2005). Face validity of the instrument was done by the project supervisor while the content validity was done by an expert panel which was composed of Maternal and Child Health Nursing experts at the Nursing Department, Ondo State Ministry of Health. The expert panel was asked to make judgement on the relevance of the content of the instrument along a continuum. The relevance was rated on a 4-point scale as follows: $1=$ not relevant; $2=$ somewhat relevant; $3=$ quite relevant and $4=$ relevant. The Item Content Validity Index (I-CVI) was determined by dividing the number of experts giving a rating of 3 or 4 , by the number of experts - that is, the proportion in agreement about relevance (Polit \& Beck, 2009). The Scale Content Validity Index (S-CVI) was thereafter computed and yielded 0.87. According to Polit and Beck (2009), an S-CVI of 0.87 establishes an excellent validity. All respondents completed the same questionnaire individually, the researcher also ensured that the retrieved Partograph forms were true copies of the ones utilized by the midwives under consideration. A statistician was consulted during questionnaire design and data analysis to eliminate data interpretation error.

\section{Reliability of the Research Instrument}

The reliability of the instrument was done through the test retest method. The instrument was administered to the respondents on two different occasions in an interval of two (2) weeks. The data obtained was subjected to the Pearson Product Moment Correlation analysis which yielded a reliability of $r=0.89$. Pearson Product Moment Correlation Coefficient $(\mathrm{r}) \geq 0.65$ indicates strong reliability, which makes the instrument acceptable.

\section{Method of Data Analysis}

The researcher checked the instrument for completeness, it was entered into a spreadsheet and checked for errors, data were collated, coded and analyzed using Statistical Package for Social Science (SPSS) version 23. To assess the levels of knowledge on the partograph used, the level of knowledge was graded into "poor knowledge" ( $\leq$ Mean Score $=15.2)$ and "Good knowledge" ( $\geq$ Mean Score 15.2). Also, to determine the levels of utilization, there were 14 items with a total score of 28 . The utilization level was categorized as good and poor utilization. The Mean score was 14.29, any score below the mean of 14.29 indicated good utilization, while the score above the mean of 14.29 indicated poor utilization. Pearson's correlation analysis was conducted to determine the relationship between the independent variable (midwives years of experience in obstetrics and gynaecology department) and the dependent variable (midwives knowledge of partograph in the management of labour, the chi-square result revealed that there 
is a significant relationship between the midwives years of work experience and their level of knowledge on partograph in the selected health facilities in Akure South Local Government Area, Ondo State.

\section{FINDINGS}

\section{Midwives Level of Knowledge on Partograph}

Midwives' knowledge of partograph showed that the majority 119 (59.5percent) of the midwives had good knowledge of partograph. Details are shown in Table 3

Table 3: Midwives Level of Knowledge on Partograph

\begin{tabular}{llll}
\hline Level of & Knowledge & Frequency (n) 200 & Percentage (\%) \\
\hline Poor & Knowledge & 81 & 40.5 \\
Good & Knowledge & 119 & 59.5 \\
\hline
\end{tabular}

\section{Partograph Utilization Rate}

Data obtained showed that the annual partograph utilization rate was highest in the tertiary facility (UNIMEDTH - 63.0\%), followed by the secondary (MCHA - 51.8\%) and primary (CHCA $-41.3 \%$ ) facilities respectively. However, the total annual partograph utilization rate was $54.4 \%$. Details are shown in Table 4 :

Table 4: Annual Record of Rate of utilization of Partograph among Midwives $(N=200)$ Between July 2018 - June 2019.

\begin{tabular}{lcccc}
\hline Utilization Record & Total & UNIMEDTH & MCHA & CHCA \\
\hline $\begin{array}{l}\text { Annual } \\
\text { Child Delivery }\end{array}$ & 4628 & 1730 & 2267 & 685 \\
Number of Partograph used & 2548 & 1090 & 1175 & 283 \\
$\begin{array}{l}\text { Annual Partograph utilization } \\
\text { Rate }\end{array}$ & $54.4 \%$ & $63.0 \%$ & $51.8 \%$ & $41.3 \%$ \\
\end{tabular}

Key: UNIMEDTH - University of Medical Sciences Teaching Hospital Akure MCHA Mother and Child Hospital Akure CHCA — Comprehensive Health Centre Arakale Akure 


\section{Utilization Levels of Partograph}

Table 5 shows the distribution of the utilization of partograph among the three levels of healthcare. It shows that 62(62\%) represents good utilization of partograph from UNIMEDTH, while $38(38 \%)$ had poor utilization, from MCHA 46(59\%) had good utilization, while 32(41\%) had poor use, and all the respondents $22(100 \%)$ in CHCA had poor utilization.

Table 5: Utilization levels of Partograph between the Healthcare Facilities.

\begin{tabular}{lll}
\hline Level of Utilization & Poor Utilization & Good Utilization \\
\hline UNIMEDTH & $38(38 \%)$ & $62(62 \%)$ \\
MCHA & $32(41 \%)$ & $46(59 \%)$ \\
CHCA & $22(100 \%)$ & $0(0 \%)$ \\
Total & $\mathbf{9 2}(\mathbf{1 0 0 \%})$ & $\mathbf{1 0 8}(\mathbf{1 0 0 \%})$ \\
\hline
\end{tabular}

\section{Hypothesis}

$\mathbf{H}_{\mathbf{0 1}}$ There is no significant relationship between respondents' years of work experience and their knowledge of Partograph in selected health facilities in Akure, Ondo State.

The chi-square test of relationship showed that a significant relationship exists between years of work experience and the midwives' level of knowledge on partograph. The P-values $(\mathrm{p}=$ 0.01 ) was less than the 0.05 alpha level of significance. The null hypothesis is rejected. Details are as shown in Table 6

Table 6: Chi-square Test of Relationship between Years of Work Experience and Level of Knowledge on partograph among Midwives

\begin{tabular}{|l|l|l|l|l|}
\hline \multicolumn{3}{l}{ Level of knowledge } & \\
\hline $\begin{array}{l}\text { Years of } \\
\text { experience }\end{array}$ & Poor knowledge & Good Knowledge & & \\
\hline $0-5$ years & 14 & 45 & 59 & $\mathrm{X} 2=11.6$ \\
$6-10$ years & 34 & 31 & 65 & $\mathrm{Df}=3$ \\
$11-15$ years & 20 & 22 & 42 & $\mathrm{P}=0.01$ \\
$16-20$ years & 13 & 21 & 34 & \\
\hline Total & $\mathbf{8 1}$ & $\mathbf{2 0 0}$ & \\
\hline
\end{tabular}

$\mathbf{H o}_{2}$ There is no significant difference in the utilization of partograph in the management of labour among midwives in the three selected health facilities in Akure, Ondo State.

Result of One-way ANOVA test to assess the differences in the utilization of partograph among midwives in the selected health facilities showed that there is a significant difference in the utilization of partograph among the midwives in the three hospitals; Mother and Child Hospital $(16.33 \pm 5.83, \mathrm{p}$ value $=0.01)$ and UNIMEDTH $(15.84 \pm 6.75, \mathrm{p}$ value $=0.007)$ and Comprehensive Health Center $(9.82 \pm 3.68)[\mathrm{f}=(2,97) 5.12, \mathrm{p}=0.008]$ as presented in the table below. Hence, the null hypothesis is rejected. 
Table 7: ANOVA test for there is no significant difference in the utilization of partograph among midwives in the three selected health facilities in Akure, Ondo State.

\begin{tabular}{lllll}
\hline & $\begin{array}{l}\text { Mean } \\
\text { square }\end{array}$ & F & DF & P \\
& 16.33 & & 2 & \\
\hline Mother and Child Hospital & 9.82 & 5.12 & 97 & 0.008 \\
Comprehensive Health Centre & 15.84 & & & \\
UNIMEDTH & &
\end{tabular}

Table 8: Post Hoc Tukey HSD Test

\begin{tabular}{llllll}
\hline \multicolumn{2}{l}{ Multiple Comparisons } & & & \\
\hline Selected health & Selected health & Mean & & \multicolumn{2}{l}{$95 \%$ Confidence Interval } \\
facility category & facility category & Difference & Sig & Lower Bound & Upper Bound \\
\hline Mother and Child & CHC & 6.52 & 0.007 & 1.52 & 11.51 \\
& UNIMEDTH & 0.49 & 0.92 & -2.63 & 3.62 \\
\multirow{2}{*}{ UNIMEDTH } & & & & & \\
& Mother and Child & -0.49 & 0.93 & -3.62 & 2.63 \\
& CHC & 6.02 & 0.01 & 1.15 & 10.89 \\
\hline
\end{tabular}

Furthermore, the Post Hoc Tukey HSD as seen in Table 8 showed that there is a significant difference in the management of labour between midwives in MCHA see CHCA $(p=0.007)$. Also, there is a significant difference in the utilization of partograph in the management of labour between the midwives in UNIMEDTH and CHCA $(\mathrm{p}=0.01)$. However, no significant difference was discovered in the utilization of partograph in the management of labour between midwives in MCHA and UNIMEDTH ( $\mathrm{p}=0.93$ ).

\section{DISCUSSION}

Research objective 1: to ascertain midwives' level of knowledge of partograph in the management of labour in selected health facilities in Akure Ondo State.

The findings of the study show that half of the midwives had good knowledge of partograph. Specifically, 59.5 percent of midwives had good knowledge, 40.5 percent had poor knowledge. The result suggests that midwives in the study milieu have the requisite knowledge to utilize partograph for the management of labour. In the reasoning that knowledge translates to practice, it could be inferred that the use of partograph among midwives in the study area would be high because of the level of knowledge they possess. If not so, the finding places responsibility on managers and administrators, to initiate strategies and programmes that would coordinate system translation of the enormous knowledge to practice achieving the benefits inherent in partograph use. The high level of knowledge may, however, be due to the nursing education they had attained as 72.5percent reported to have heard of partograph through educational training. The finding supports the work of Asibong et al (2014), Mathibe (2013), 
Wakgari et al (2015), and Fawole et al (2012) which reported good knowledge among many of their respondents. However, while Asibong et al (2014), Mathibe (2013)reported fair knowledge, Fawole et al (2008) and Fawole et al (2010) reported poor knowledge among their respondents, few of the respondents in this study demonstrated poor knowledge.

Research objective 2: to determine the level of utilization of partograph in management of labour amongst midwives in selected health facilities in Akure Ondo State.

The total annual partograph utilization rate among midwives was 54.4percent. Out of a total of 4628 labour cases, only 2548 were managed with partograph in the selected healthcare facilities. Findings on the levels of utilization further show that 62 (62 percent) represents good utilization of partograph from UNIMEDTH, while 38(38 percent) had poor utilization and 46 (59percent) from MCHA had good utilization, while 32(41percent) had poor use, and all the checklists 22 (100percent) had poor utilization in CHCA. The finding indicates a gap between knowledge and utilization. The level of knowledge of respondents demonstrated in this study seems not to correlate with the utilization level of partograph in CHCA. The implication is that the quality of midwifery services might gradually diminish and eventually lead to an increase in morbidity, mortality and patient dissatisfaction. It is, therefore, necessary to take relevant steps to ensure that knowledge gained while training and service is practically applied when it is required.

Considering the gap between respondents' knowledge of partograph and its use, it would not be out of place to infer that personal and institutional barriers to utilization exist. The finding, therefore, supports the identification of personal and institutional-specific barriers that tend to impede utilization. Identifying such barriers would help in determining strategies for their elimination or reduction.

A vital point to also note is the fact that utilization rate was higher at the tertiary facility (63percent) than the secondary, while the secondary (51.8percent) was higher than the primary level (41.3percent). What this means is that more mothers at the primary and secondary levels are denied the benefits of partograph during labour, while the risk level of childbirth is increased. The finding indicates the need for proper institutional monitoring to ensure maximum utilization of partograph at the primary and secondary level facilities. The higher rate of utilization at the tertiary level facility may, however, be so due to the higher number of midwives in the tertiary level facility as it is shown in the demographic data. This further indicates the employment of additional midwives to improve utilization rates. The underutilization observed in this study is like the findings of Ogwang et al (2009), Maphasha et al (2012), and Opoku and Nguah (2015), which also reported poor utilization of partograph for labour management in Rujumbura Health District in Uganda. According to Ogwang et al (2009), Maphasha et al (2012), and Opoku and Nguah (2015), the poor utilization of partograph among their respondents in a public health institution in Kwasi, Ghana was due to inadequate knowledge and skills and the shortage of midwives in their study areas.

Research objective 3: to identify the factors affecting level of utilization of partograph in the management of labour amongst midwives in selected health facilities in Akure Ondo State.

Of all the seven (7) variables that were considered, six (6) were perceived to have affected the utilization of partograph by midwives. First on the list of variables was midwives' level of confidence. Respondents acknowledged that their level of confidence affected how often they 
utilize partograph for the management of labour. In the researcher's view, not having confidence in using the partograph may be due to inadequate training on practical skills which was a cofactor the study identified to have influenced partograph use. Thus, providing adequate training and retraining programmes may help sharpen midwives' skills and offer them the needed confidence for improved utilization. The shortage of staff was the second variable perceived to have affected the utilization of partograph for labour management. The shortage of staff was reflected in the demographic data especially at the primary level where there were only 22 midwives with an annual partograph utilization rate of 41.3percent. The present study is in line with Mathibe (2013); Yisma et al (2103) and Opiah et al (2012) whose studies revealed that shortage of midwives is one of the factors that contribute to the under-utilization of partograph in Johannesburg South Africa, Addis Ababa, Ethiopia and Niger Delta Region of Nigeria

Inadequate supply of partograph forms and management policies were among factors identified to have also influenced the utilization of partograph. This is in correlation to the study of Ogwang et al., 2009, that shows that non-availability or shortage of partograph implies poor utilization of partograph in Uganda. Furthermore, Asibong et al supports the present study as revealed that the inability of hospitals' managements to produce benchmarks on the use of partograph during labour, poor managerial support regarding the procurement of necessary supplies needed during labour affects partograph utilization in Calabar, Cross Rivers State.

The findings of this present research further reveal that knowledge is a factor affecting the use of partograph which is consistent with the research reports of significant others. For instance, Engender Health (2012) and Opiah et al (2012) also identified knowledge as a barrier to utilization in Bayelsa State while Mathibe (2013), Yisma et al (2013) and Opiah et al (2012) reported a shortage of midwives as a factor affecting the use of partograph. More so, Yisma et al (2013) reported lack of training; Engender Health (2012) reported inadequate time on the job in Southern Ethiopia while Asibong et al (2009) reported inadequate supply of partograph forms in Calabar, Cross Rivers State.

Hypothesis: There is no significant relationship between respondents' years of work experience and their levels of knowledge on partograph in selected health facilities in Akure, Ondo State.

The hypothesis above was tested and showed that a significant relationship existed between years of work experience and the midwives' level of knowledge on partograph $(\mathrm{P}=0.01)$. These findings were expected and not surprising as the study of Wakgari et al 2015 in North Shoa Zone, Central Ethiopia also found out that getting on the job training and working in the hospital are significantly related to knowledge of partograph. Furthermore, hypothesis which stated that there is no significant difference in utilization of partograph among nurses in the selected health facilities in Akure, Ondo State was rejected at p value (0.008) is less than 0.05 alpha level of significance. Which is similar to the result of the study by Markos and Bogale, (2016) whose study in South East Ethiopia showed that the magnitude of partograph utilization was 70.2 percent, as being hospital staff ( $\mathrm{AOR}=17.78,95$ percent $\mathrm{CI}=2.32,135.98)$ have statistically significant association with partograph utilization. 


\section{Implication to Research and Practice}

The study revealed a gap between midwives' knowledge on partograph and its utilization levels in the primary healthcare facility which is the health care grass-root point of entry. It also identified several factors that affected the utilization of partograph for the management of labour. The implication of the study is, therefore, the immediate initiation of viable programmes including training on practical skills that would drive system translation of knowledge to practice. Institution-based strategies and resources are also needed to eliminate or reduce factors that have been identified to impede the utilization of partograph for the management of labour.

\section{CONCLUSION}

The midwives in the three selected health facilities had good knowledge of partograph as a labour monitoring tool for implementing safe motherhood. However, the utilization level was poor at the CHCA (primary health care facility).Considering the gap between the midwives knowledge of partograph and its use despite the years of work experience at the obstetric and the gynaecology department, it would not be out of place to infer that personal and institutional barriers to utilization exist. Although, lack of confidence, shortage of staff, inadequate supply of partograph form, and inadequate training on the use of partograph were identified as major factors affecting the utilization. There is a need for continuous in- service training and retraining programme on partograph utilization, proper monitoring is important, and more midwives should be employed and deployed to the primary healthcare facilities to reduce

\section{RECOMMENDATIONS}

Based on the findings of the study, it is recommended that the head of nursing unit in all health facilities should encourage midwives and motivate them to gain confidence when using the partograph in managing women in labour by training and retraining them on evidence based practice on the use of partograph. It is recommended that the Government should employ and post midwives to the primary and secondary level facilities so that there will be more midwives attending to women in labour. The findings of this study also necessitate the need for a review of managerial policies on the midwifery job descriptions to accommodate optimal use of partograph across the levels of healthcare delivery. Midwives at the primary facilities should be trained and motivated on adequate use of the partograph in the management of labour.

Furthermore, stakeholders should have a regular audit to monitor and evaluate the effective use of partograph within the primary health facilities. Finally, practical skills training and retraining programmes should be provided to enhance midwives' skills and boost their confidence for improved use of partograph maternal mortality and neonatal deaths at the grass root level of the health care system. 


\section{REFERENCE}

Abebe, F. Birhanu, D. Awoke, W. \& Ejigu, T. (2013). Assessment of knowledge and utilization of the partograph among health professionals in Amhara region, Ethiopia, Journal of Clinical Medicine Research. 2(2): 1-17 doi: 10.11648/j-sjcm

Asibong,U.Okokon, I. B. Agan, T. U., Oku, A. Opiah, M., Essien, E. J. \& Monjok, E. (2014). The use of the partograph in labor monitoring: A cross-sectional study among obstetric caregivers in General Hospital, Calabar, Cross River State, Nigeria. International Journal of Women's Health, 6, 873-880. doi:10.2147/IJWH.S49188

Badran, Ibrahim G. (1995). Knowledge, attitude and practice are the three pillars of excellence and wisdom: a place in the medical profession. EMHJ - Eastern Mediterranean Health Journal, 1 (1), 8-16, 1995 https://apps.who.int/iris/handle/10665/116905

Bedwell,C. Levin, K. Pett, C.\& Lavender,D.T (2017) A realist review of the partograph:when and how does it work for labour monitoring?.BMC Pregnancy Childbirth 17,31.https//doi org/10.1186/s12884-016-1213-4

Bosse, G. Massawe, S. \& Jahn, A. (2002). The partograph in daily practice; it's quality that matters, International Journal of Gynaecology and Obstetrics;77(3)243-244. doi: 10.1016/s0020-7292(02)00004-8 pmid: 12065136

Burns N, Grove S (2005)Practice of Nursing Research;Appraisal,synthesis, and generation of evidence,Philadelphia, Saunders elsevier.

Dolea, C. \& Abouzahr, C. (2003). Global burden of obstructed labour in the year 2000. Who, $1-17$.

Fawole, A. O. Hunyinbo, K. I. \& Adekanle, D. A. (2008). Knowledge and utilization of the partograph among obstetric care givers in south west Nigeria. African Journal of Reproductive Health, 12(1), 22-29. doi:10.2307/25470629

Fawole,A.O,Adekanle, D.A,Hunyinbo,K.I (2010) "Utilization of the partograph in primary health care facilities in southwestern Nigeria,"Nigerian Journal of Clinical Practice,13(2),pp2-5,PMID 20499756.

FMOH,Maternal Death Surveillance and Response (MDSR),Technical Guideline,Addis Ababa,Ethiopia,2012.

Lavender, T. Omoni, G. Lee, K. Wakasiaka, S. Watiti, J. \& Mathai, M. (2011). Students' experiences of using the partograph in Kenyan labour wards. African Journal of Midwifery and Women's Health, 5, 117-122. doi:10.12968/ajmw.2011.5.3.117

Maphashaa, O. M. Govender, I. Motloba, D. P. \& Barua, C. (2017). Use of the partogram by doctors and midwives at Odi District Hospital, Gauteng, South Africa. South African Family Practice, 59(2), 82-86.

Ogwang, S. Karyabakabo, Z. \& Rutebemberwa, E. (2009). Assessment of Partograph Use during labour in Rujumbura Health Sub District, Uganda. African Health Science. 9:527534.

Opiah, M. M. Ofi, A. B. Essien, E. J. \& Monjok, E. (2012). Knowledge and utilization of the partograph among midwives in the Niger Delta Region of Nigeria. African Journal of Reproductive Health, 16(1), 125-132.

Opoku, B. K., \& Nguah, S. B. (2015). Utilization of the modified WHO partograph in assessing the progress of labour in a metropolitan area in Ghana. Research Journal of Women's Health, 2(1), 2. doi:10.7243/2054-9865-2-2

Polit,O.F \& Beck,C.T (2009) Nursing research.Generating and assessing evidence for nursing practice $\left(8^{\text {th }}\right.$ edition $)$ Philadelphia Wolters Klower/ Lippincott Williams \& wilkins. 
Rakotonirina, J. Randrianantenainjatovo, C. Vololonarivelo, B. Dorasse, R. Rakotomanga, J. \& Rakotovao, A. (2013). Assessment of the use of partographs in the region of Analamanga. International Journal of Reproduction, Contraception, Obstetrics and Gynecology, 2(3), 257-262. doi:10.5455/2320-1770.ijrcog20130901

Sama, C.-B. Takah, N. F. Danwe, V. K. Melo, U. F. Dingana, T. N. \& Angwafo, F. F (2017). Knowledge and utilization of the partograph: A cross-sectional survey among obstetric care providers in urban referral public health institutions in northwest and southwest Cameroon. PloS One, 12(2), e0172860. doi:10.1371/journal.pone.0172860

Tadesse, E. (2012). The partograph for the prevention of obstructed labour. Science Journal of Clinical Medicine.8(12): 20-31.

Wakgari, N. Amano, A. Berta, M. \& Tessema, G. A. (2015). Partograph utilization and associated factors among obstetric care providers in North Shoa Zone, Central Ethiopia: a cross sectional study. African Health Sciences, 15(2), 552-559. doi:10.4314/ahs.v15i2.30

WHO, World Health Organisation, (2011). Fact Sheet, Maternal, Newborn, Child and Adolescent Health. 65(2): 203-205.

World Health Organization (2016) SDG Health and HealthRelated Targets, Geneva http;2015//www.who.int/gho/publications/mdgs-sdgs/en/,accessed 5 February 2019.

World Health Organization (WHO), (2015). Trends in Maternal Mortality: 1990 to 2015. Geneva, Switzerland. Estimates and the World Bank. Geneva:

Yisma, E. Dessalegn, B. Astatkie, A. \& Fesseha, N. (2013). Knowledge and utilization of partograph among obstetric care givers in public health institutions of Addis Ababa, Ethiopia. BMC Pregnancy and Childbirth, 13, 17. doi:10.1186/1471-2393-13-17

Zelellw, D. A. \& Tegegne T. K. (2018): Questionnaire on partograph knowledge and utilization. PLOS ONE. Journal contribution. https://doi.org/10.1371/journal contribution 